\title{
The parasitic flagellates Trichomonas vaginalis and Tritrichomonas foetus produce indole and dimethyl disulphide: direct characterization by membrane inlet tandem mass spectrometry
}

\author{
David Lloyd, ${ }^{*} \dagger$ Frants R. LaURitsen and Hans Degn \\ Biokemisk Institut, Odense Universitet, DK-5230, Campusvej 55, Odense M, Denmark \\ (Received 17 January 1991; revised 25 March 1991; accepted 9 April 1991)
}

\begin{abstract}
The use of a membrane inlet triple quadrupole mass spectrometer revealed indole as an end product in the growth medium of cultures of the cattle parasite Tritrichomonas foetus and the human parasite Trichomonas vaginalis: formation of indole is enhanced in the presence of added tryptophan. Two different clinical isolates of Trich. vaginalis also produce dimethyl disulphide. Electron impact ionization yielded complex fragmentation mixtures, but the facility for analysis of daughter ions enabled unequivocal assignments. Chemical ionization gave $[\mathrm{M}+1]^{+}$ species, and tandem mass spectrometry produced identification through daughter ions. The method provides a rapid single-step procedure for the characterization of microbial products without the need for preliminary separation and derivatization.
\end{abstract}

\section{Introduction}

The application of non-invasive physical methods to investigations of the metabolism of growing cultures of organisms or cells often provides new insights (Lloyd $e t$ al., 1989): a recent example is the use of ${ }^{13} \mathrm{C}$-NMR for the identification of glycerol as a major fermentation product of Trichomonas vaginalis (Chapman et al., 1985). In the case of clinically important organisms, the identification of unusual metabolic characteristics may provide new avenues for chemotherapeutic attack (Linstead \& Cranshaw, 1983; Thong \& Coombs, 1987b). Ishiguro (1985) has used GC-MS to identify gaseous trichomonad products. Here we describe the use of a membrane inlet triple quadrupole mass spectrometer for the rapid identification of indole and dimethyl disulphide as unexpected metabolic end-products of trichomonads.

\section{Methods}

Maintenance and growth of organisms. Tritrichomonas foetus and Trichomonas vaginalis (strains 1910 and $\mathrm{UCH}-1$ ) were obtained from $\mathrm{Dr}$ Alex Yule and Mr Bob Spice of the London School of Hygiene and Tropical Medicine. Cultures were frozen after adding $10 \%(\mathrm{v} / \mathrm{v})$ dimethylsulphoxide by cooling in a cryostat at $1{ }^{\circ} \mathrm{C}$ per min and stored

† Present Address: Microbiology Group, (PABIO), University of Wales College of Cardiff, PO Box 915, Cardiff CF1 3TL, Wales, UK. at $-80{ }^{\circ} \mathrm{C}$. They were grown at $37^{\circ} \mathrm{C}$ in screw-capped tubes $(10 \mathrm{ml})$ or bottles $(100 \mathrm{ml})$ in modified Diamond's (1957) medium containing ascorbate, cysteine, and $10 \%(\mathrm{v} / \mathrm{v})$ heat-inactivated horse serum: organisms were subcultured daily. Recovery of frozen organisms was followed by serial subculture: at least 12 transfers $(0 \cdot 1 \mathrm{ml}$ inocula in $10 \mathrm{ml}$ media) preceded analyses of product formation. Organisms were counted in a modified Fuchs-Rosenthal haemocytometer (depth $0.2 \mathrm{~mm}, 1 / 16 \mathrm{~mm}^{2}$ ).

Treatment of samples. Culture supernatants were prepared by centrifugation at $4^{\circ} \mathrm{C}$ in a Haraeus bench centrifuge at $1000 \mathrm{~g}$ for $2 \mathrm{~min}$, carefully decanted and immediately frozen at $-18^{\circ} \mathrm{C}$. Mass spectrometric analyses were performed directly on undiluted samples $(3 \mathrm{ml})$.

Tandem mass spectrometry. The membrane inlet triple quadrupole mass spectrometer was based on the design of Yost \& Enke (1979) and has been previously described in detail (Lauritsen et al., 1990): it was constructed in collaboration with VG Quadrupoles, Cheshire, UK specially for process control using a membrane inlet. A VG 20-253T dual chemical ionization/electron impact ion source and quadrupole analysers (125 mm VG SX200 rods) were enclosed in two differentiallypumped stainless steel chambers evacuated by two $240 \mathrm{ll}^{-1}$ turbomolecular pumps (Balzers, Lichtenstein). Edwards PE 505 Penning gauges were used to measure total pressure values. Three synchronized VG SX200 RF generators $(0-880 \mathrm{~V})$ were used. Axial ion energy was variable between -50 and $+50 \mathrm{~V}$ in the collision cell, and between +12 and $-12 \mathrm{~V}$ in the mass-analysing quadrupole. Mass scanning used a PC with 14-bit $\mathrm{A} / \mathrm{D}$ and D/A converters. Scan modes enabled computer-controlled scanning of first quadrupole only, third quadrupole only, or of first quadrupole with the third quadrupole delayed, enabling acquisition of normal mass spectra, daughter ion, parent ion or neutral loss spectra.

The thermostatted stainless steel reaction vessel ( $4 \mathrm{ml}$ total volume) was interfaced with the vacuum system at a point as close as possible to 
the ion source. The liquid sample was separated from the vacuum by a $25 \mu$ m-thick silicone rubber membrane (Radiometer, Copenhagen) supported on a stainless steel porous plug. Stirring was by a verticallymounted impellor in order to minimize effects of the unstirred liquid layer on the membrane.

\section{Results}

Volatile compounds accumulating in the growth medium of Trit. foetus

Electron impact ionization mass spectrometry (EI-MS) of those substances which are volatile at $\mathrm{pH} 6.2$ and are able to penetrate the $25 \mu \mathrm{m}$-thick silicone membrane indicates that uninoculated Diamond's medium contains a variety of such compounds, and the fragmentation pattern is complex, with major maxima at $m / z=58,128$, $72,105,106,57,55,77,56$ and 64 (Fig. 1 a). After growth of Trit. foetus for $2 \mathrm{~d}$ (the late exponential phase of growth, $4 \times 10^{6}$ organisms $\mathrm{ml}^{-1}$ ) a mass spectrum acquired under identical conditions (Fig. $1 b$ ) indicated that the volatiles giving rise to this fragmentation pattern had been utilized. The major components of the EI-MS spectrum were now at $m / z=55,117,56,70,57,71,53$, $58,89,90$ and 88. At stationary phase (Fig. $1 c, 6 \times 10^{6}$ organisms $\mathrm{ml}^{-1}$ ) the intensities of peaks at $\mathrm{m} / \mathrm{z} 117,70$, $71,89,90$ and 88 had increased about two-fold over the corresponding features at $2 \mathrm{~d}$, whereas those at $m / z=55$ and 80 had declined.

When methane was used as reaction gas for chemical ionization (CI) a low-intensity background of symmetric sets of peaks is observed at low masses $(m / z<150)$. This background is caused by gas-phase reactions between reaction ions and the reaction gas itself and by impurities in the reaction gas (grade 3.5). Further, the CI-MS spectrum of the stationary phase sample (Fig. $2 a$ ) clearly shows two peaks at $m / z 118$ and 117. A daughter ion spectrum of the $[M+1]^{+}$parent ion $\mathrm{m} / \mathrm{z} 118$ gave fragments at $m / z 91$ and 65 (Fig. $2 b$ ). Table 1 summarizes these data.

Fig. $3 a$ shows the EI-MS spectrum from a stationary phase culture of Trich. vaginalis $1910\left(3 \times 10^{6}\right.$ organisms $\mathrm{ml}^{-1}$ ). Peaks at mass 117,90 and 89 are present, but in this case the dominant features of the mass spectrum are at 94 and 79. CI-MS showed peaks at $m / z 95,117$ and 118. A daughter-ion mass spectrum (EI) of the species at mass 94 gave fragments at 79 and 61 (Fig. $3 b$ ).

Comparison of the data with 'Eight Peak Index of Mass Spectra' (Anon, 1983) entries at $m / z 117$ and 94 indicates an excellent match with the fragmentation patterns for indole and dimethyl disulphide respectively (Table 2). Standard spectra of solutions of indole and dimethyl disulphide (Figs $4 a$ and $4 b$, respectively) show excellent agreement with indole peaks (117, 90 and 89)
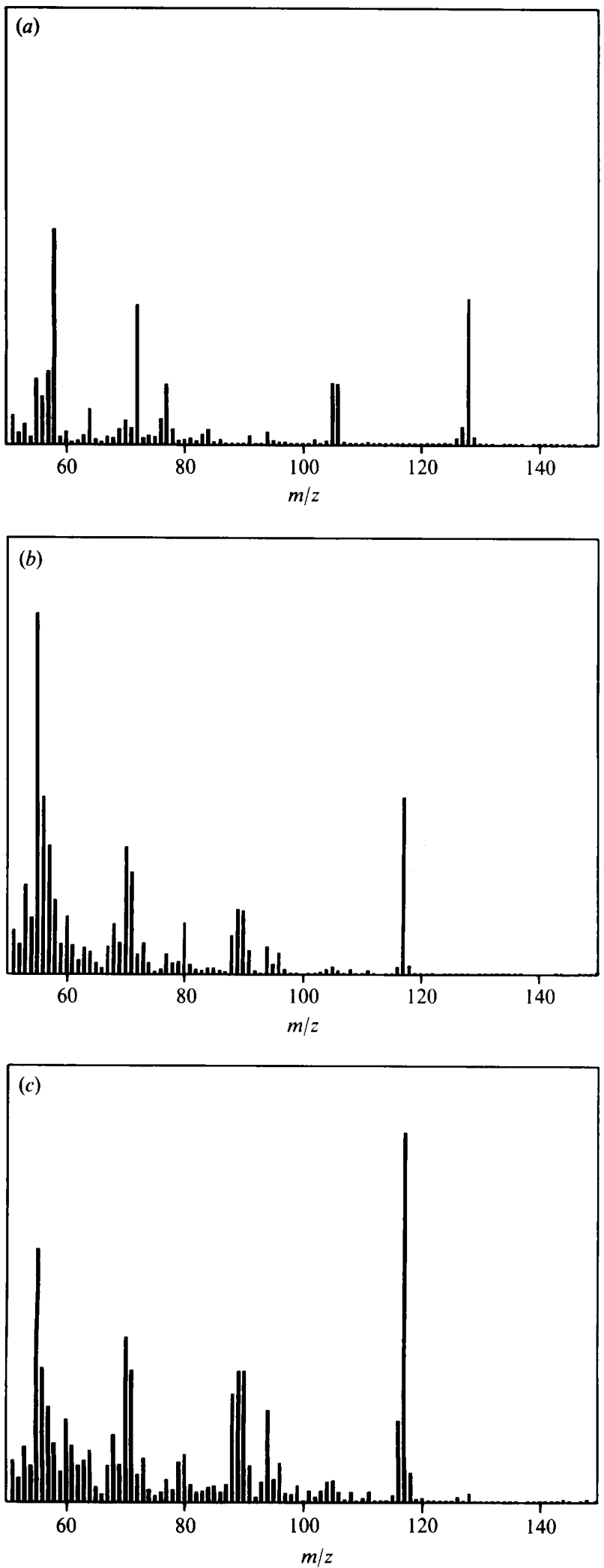

Fig. 1. Changes in ion fragments of volatile components in the growth medium of Tritrichomonas foetus. Mass spectra obtained after electron impact ionization of components from (a) uninoculated medium, (b) $2 \mathrm{~d}$ culture, and (c) $4 \mathrm{~d}$ culture. 

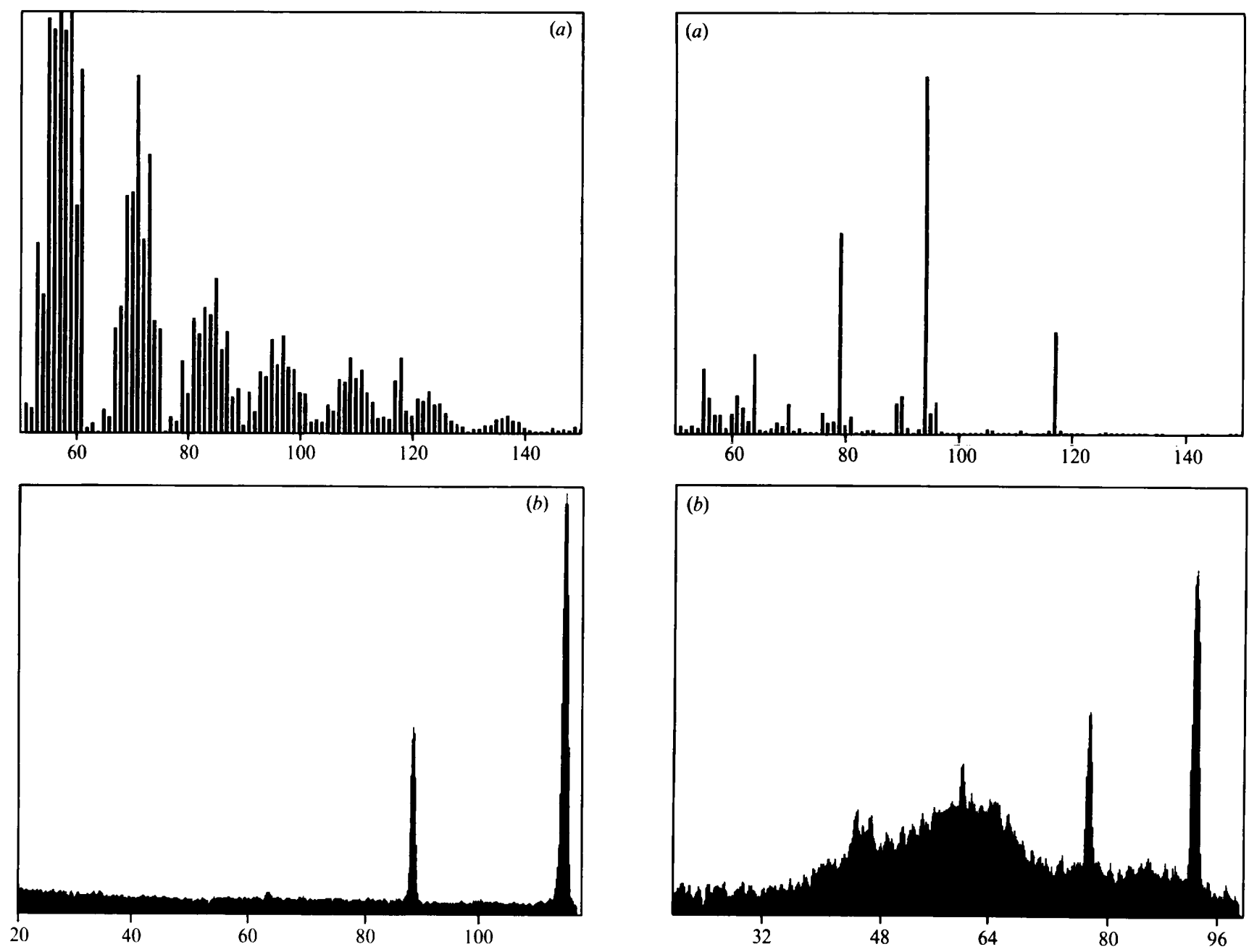

Fig. 2. Tandem mass spectrometry of volatile components in the growth medium of Tritrichomonas foetus. (a) Chemical ionization mass spectrometry showing parent $[\mathrm{M}+1]^{+}$superimposed on background spectrum of $\mathrm{CH}_{4}$. (b) Tandem mass spectrometry of the parent ion at $m / z=118$.

Table 1. Mass spectrometric analyses of volatile components in the growth medium of Tritrichomonas foetus

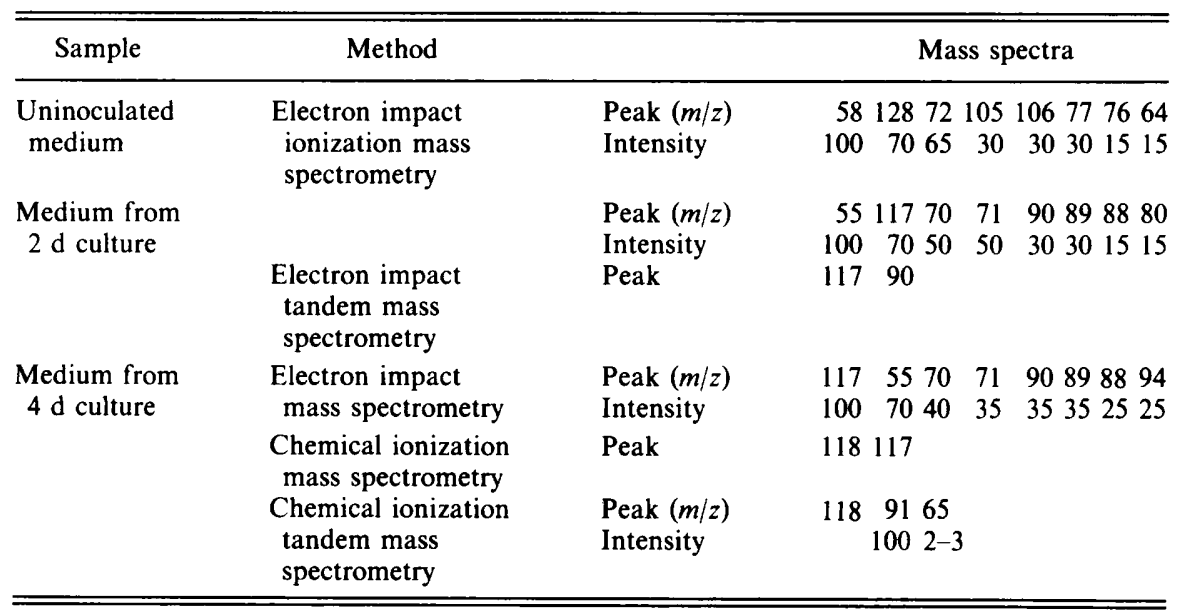



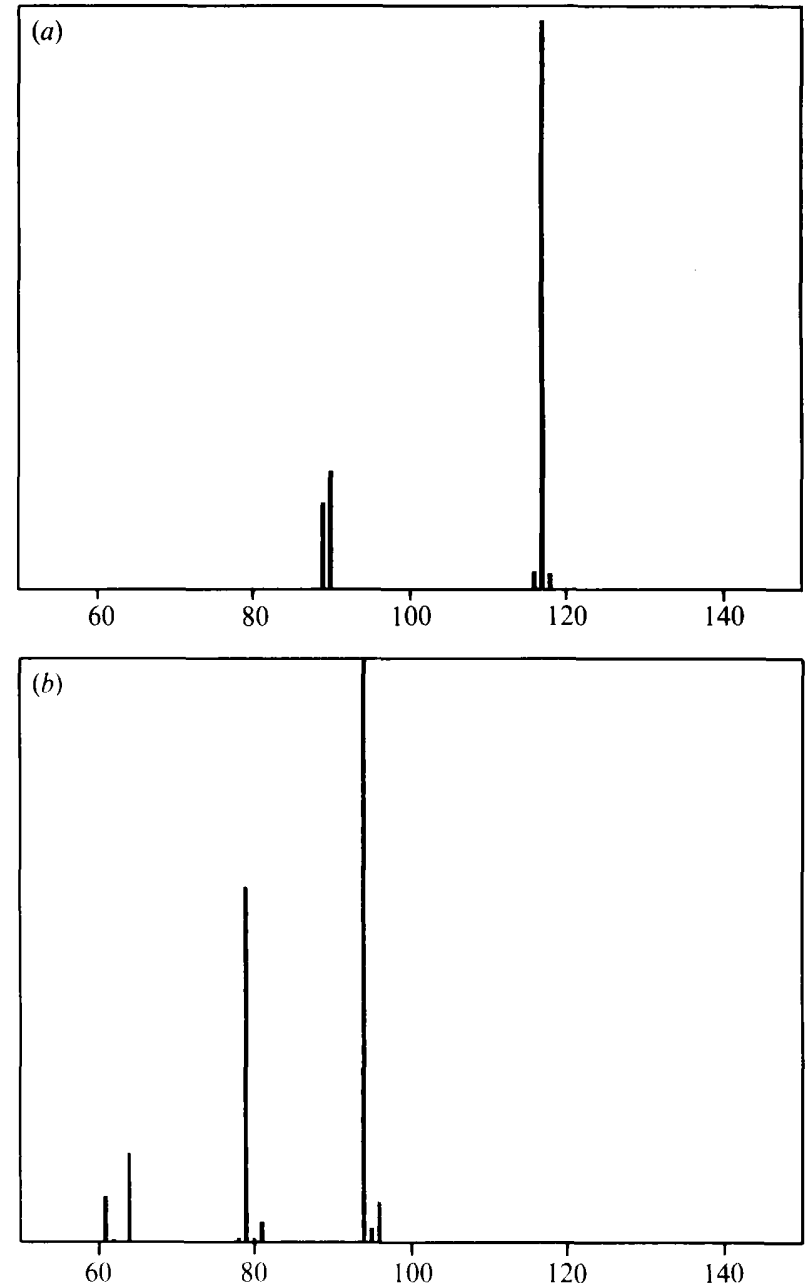

Fig. 4. Electron impact ionization mass spectrum of $(a)$ indole and $(b)$ dimethyl disulphide.

Table 2. Mass spectra of indole and dimethyl disulphide after electron impact ionization (Eight Peak Index)

\begin{tabular}{lrrrrrrr}
\hline \hline \multicolumn{7}{c}{ Indole $\mathrm{C}_{8} \mathrm{H}_{7} \mathrm{~N}=117$} \\
\hline Peak $(m / z)$ & 117 & 90 & 89 & 63 & 39 & 116 & 118 \\
Intensity & 100 & 40 & 24 & 14 & 9 & 8 & 8 \\
\hline \multicolumn{7}{c}{ Dimethyl disulphide $\mathrm{C}_{2} \mathrm{H}_{6} \mathrm{~S}_{2}=94$} \\
\hline Peak $(m / z)$ & 46 & 79 & 45 & 94 & 49 & 44 & 61 \\
Intensity & 100 & 99 & 99 & 98 & 71 & 36 & 33 \\
\hline \hline
\end{tabular}

found in both cultures and with dimethyl disulphide peaks $(m / z$ 94, 79, 64 and 61) found in Trich. vaginalis strain 1910. Standard daughter ion mass spectra of the molecular ions $[\mathrm{M}+1]^{+}$(indole; $\mathrm{CI}$ ) and $[\mathrm{M}]^{+}$(dimethyl disulphide; EI) showed excellent agreement with the spectra observed from these ions from the cultures. The concentrations of indole and dimethyl disulphide in the 4-d-old sample of Trich. vaginalis strain 1910 were estimated to be $20 \mu \mathrm{M}$ and $4 \mu \mathrm{M}$, respectively. Trich. vaginalis strain $\mathrm{UCH}-1$ gave similar results to those shown for strain 1910: when grown for $24 \mathrm{~h}$ in the presence of 5 mM-tryptophan, the former strain gave an indole/dimethyl disulphide ratio twice that of organisms grown in its absence.

\section{Discussion}

Both indole and dimethyl disulphide (Table 2) are known end-products of amino acid catabolism. The bacterial conversion of tryptophan to indole has been known since the work of Hopkins \& Cole (1903); tryptophanase, a pyridoxal-phosphate-requiring enzyme, cleaves tryptophan to give indole, pyruvate and ammonia (Wood et al., 1947; Gooder \& Happold, 1954). This reaction can occur anaerobically, and aminoacrylic acid is the immediate precursor of pyruvate and ammonia.

The major nitrogenous excretory product of many parasitic protozoa is ammonia (Gutteridge \& Coombs, 1977). In some trypanosomatids, tryptophan- $\alpha$-ketoglutarate aminotransferase yields glutamate and indolepyruvate; the latter compound is then reduced to indolelactate or decarboxylated to indole-acetate and tryptophol (Stibbs \& Steed, 1975). Trichomonads have a great capacity to digest proteins (Lockwood et al., 1987; Lockwood \& Coombs, 1989); although critical experiments have not been performed, it seems likely that they utilize amino acids as sources of energy when available in excess, especially if carbohydrates are not available. In the experiments reported here, the growing organisms have a plentiful supply of maltose, and the trace amounts of indole detected may not be representative of the full potential for utilization of the tryptophan degrading pathway that would be only manifest under conditions of carbohydrate starvation. No detailed studies of tryptophan catabolism in trichomonads have been reported; that indole accumulation in the growth medium is enhanced when organisms were incubated in the presence of added tryptophan, indicates a percursorproduct relationship.

By comparison, the interconversions involved in the trichomonad metabolism of sulphur-containing amino acids are much better understood (Thong et al., $1987 a, b$; Thong et al., 1987). Methionine, required for the production of $S$-adenosyl methionine, the central metabolite for transmethylation reactions, can be rapidly hydrolysed by methionine $\gamma$-lyase to methanol in rumen ciliate protozoa (Merricks \& Salisbury, 1973, 1974; Onodera \& Migita, 1985) and in a pseudomonad (Tanaka et al., 1985). Methanethiol, first detected as a product of trichomonad metabolism by Gobert et al. (1971), is easily 
autoxidized in solution to dimethyl disulphide. Our results confirm those of Thong \& Coombs (1987b) who showed that of five trichomonad-like organisms, only Trich. vaginalis produces significant quantities of methanethiol.

The rapid and direct identification of metabolic endproducts without prior purification or derivatization described here provides an example of the usefulness of membrane inlet tandem mass spectrometry in the recognition of previously unsuspected metabolic pathways and transformations.

This work was supported by the Centre for Food and Process Biotechnology, The Technical University, Copenhagen.

\section{References}

ANON (1983). Eight Peak Index of Mass Spectra, The Mass Spectrometry Data Centre, The Royal Society of Chemistry, Nottingham.

Chapman, A., Linstead, D. J., Lloyd, D. \& Williams, J. (1985). ${ }^{13}$ CNMR reveals glycerol as an unexpected major metabolite of the protozoan parasite Trichomonas vaginalis. FEBS Letters 191, 287292.

Diamond, L. S. (1957). The establishment of various trichomonads of animals and man in axenic cultures. Journal of Parasitology 43, 488490.

Gobert, N., Chaigneau, M. \& Savel, J. (1971). Etude des gaz libérés au cours de la culture on anaérobiose de Trichomonas vaginalis. Comptes Rendus des Séances de la Société de Biologie 165, 276-282.

GoODER, H. \& HAPPOLD, F. C. (1954). The tryptophanase-tryptophan reaction. The nature of the enzyme-coenzyme-substrate complex. Biochemical Journal 57, 369-374.

GuTTERIDGE, W. E. \& COOMBS, G. H. (1977). Biochemistry of Parasitic Protozoa pp. 98-99. London: Macmillan Press.

Hopkins, F. G. \& Cole, S. W. (1903). The constitution of tryptophan and the action of bacteria upon it. A contribution to the chemistry of proteids Part II. Journal of Physiology 29, 451-466.

IsHIGURO, T. (1985). Gas chromatographic-mass spectrometric analysis of gases produced by Trichomonas vaginalis in vitro. Acta Obstetrica et Gynaecologica Japanica 37, 1097-1102.

Lauritsen, F. R., BohatKa, S. \& Degn, H. (1990). A membrane inlet tandem mass spectrometer for continuous monitoring of volatile organic compounds. Rapid Communications in Mass Spectrometry 4, 401-404.
Linstead, D. \& Cranshaw, M. (1983). The pathway of arginine catabolism in the parasitic flagellate Trichomonas vaginalis. Molecular and Biochemical Parasitology 8, 241-252.

Lloyd, D., Yarlett, N., Hillman, K., Paget, T. A., Chapman, A., Ellis, J. E. \& Williams, A. G. (1989). Oxygen and aerotolerant protozoa: studies using non-invasive techniques. In Biochemistry and Molecular Biology of 'Anaerobic' Protozoa, pp. 1-21. Edited by D. Lloyd, G. H. Coombs \& T. A. Paget. Chur, Switzerland: Harwood Academic.

LockWOoD, B. C. \& CoOMBs, G. H. (1989). The catabolism of amino acids by Trichomonas vaginalis. In Biochemistry and Molecular Biology of 'Anaerobic' Protozoa, pp. 93-111. Edited by D. Lloyd, G. H. Coombs \& T. A. Paget. Chur, Switzerland: Harwood Academic.

LockWOoD, B. C., North, M. J., SCOTT, K. I., Bremner, A. F. \& CoOmbs, G. H. (1987). The use of a highly sensitive electrophoretic method to compare the proteinases of trichomonads. Molecular and Biochemical Parasitology 24, 89-95.

MerRicks, D. L. \& Salisbury, R. L. (1973). The involvement of vitamin $\mathrm{B}_{6}$ in dethiomethylation by rumen microorganisms in vitro. Journal of Animal Science 37, 368-372.

MERRICKS, D. L. \& SALISBURY, R. L. (1974). Involvement of vitamin B 6 in dethiomethylation by rumen microorganisms. Applied Microbiology 28, 106-111.

ONODERA, R. \& Migita, R. (1985). Metabolism of threonine, methionine and related compounds in mixed rumen ciliate protozoa. Journal of Protozoology 32, 326-330.

StiBbs, H. H. \& STEED, J. R. (1975). Further studies on the metabolism of tryptophan in Trypanosoma brucei gambiense: Cofactors, inhibitors and end-products. Experientia 31, 274-277.

TANAKA, H., EsaKI, N. \& SodA, K. (1977). Properties of L-methionine$\gamma$-lyase from Pseudomonas ovalis. Biochemistry 16, 100-106.

THONG, K. W. \& COOMBS, G. H. (1987a). Trichomonas species: homocysteine desulphurase and serine sulphydrase activities. Experimental Parasitology 63, 143-151.

THONG, K. W. \& CoOMBs, G. H. (1987b). The effects of inhibitors of sulphur containing amino acid metabolism on the growth of Trichomonas vaginalis in vitro. Journal of Antimicrobial Chemotherapy 19, 429-437.

Thong, K. W., CoOmbs, G. H. \& Sanderson, B. E. (1987). LMethionine catabolism in trichomonads. Molecular and Biochemical Parasitology 23, 223-231.

WOOD, W. A., GunSALuS, I. C. \& UMBreit, W. W. (1947). Function of pyridoxal phosphate: resolution and purification of the tryptophanase enzyme of Escherichia coli. Journal of Biological Chemistry 170, 313-321.

Yost, R. A. \& ENKE, C. G. (1979). Triple quadrupole mass spectrometry for direct mixture analysis and structure elucidation. Analytical Chemistry 51, 1251A-1264A. 